\title{
IASI NG instrument performance status
}

\section{E. Baldit, A. Penquer, F. Bernard, F. Bermudo}

E. Baldit, A. Penquer, F. Bernard, F. Bermudo, "IASI NG instrument performance status," Proc. SPIE 11852, International Conference on Space Optics - ICSO 2020, 1185227 (11 June 2021); doi: 10.1117/12.2599628

SPIE Event: International Conference on Space Optics - ICSO 2021, 2021, Online Only 


\section{International Conference on Space Optics-ICSO 2020}

Virtual Conference

30 March-2 April 2021

Edited by Bruno Cugny, Zoran Sodnik, and Nikos Karafolas
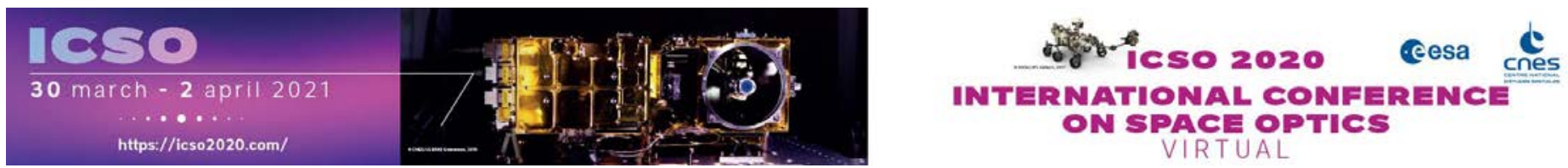

\section{IASI NG instrument performance status}

\section{Cesa isopmeatians ecnes}

International Conference on Space Optics - ICSO 2020, edited by Bruno Cugny, Zoran Sodnik, Nikos Karafolas, Proc. of SPIE Vol. 11852, 1185227 - (c) 2021 ESA and CNES

CCC code: $0277-786 X / 21 / \$ 21 \cdot$ doi: $10.1117 / 12.2599628$ 


\title{
IASI NG instrument performance status
}

\author{
E. Baldit*a, A. Penquer ${ }^{\mathrm{a}}$, F. Bernard ${ }^{\mathrm{a}}$, F. Bermudo ${ }^{\mathrm{a}}$

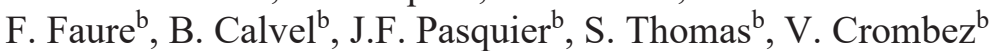 \\ ${ }^{a}$ CNES 18 Av. Edouard Belin, 31401 Toulouse Cedex 9, France \\ ${ }^{\mathrm{b}}$ Airbus Defence and Space, Rue des Cosmonautes, 31401 Toulouse, France \\ *elisa.baldit@,cnes.fr $(+33) 561282106$
}

\begin{abstract}
Developed by Airbus Defense and Space under CNES overall responsibility in partnership with Eumetsat, the Infrared Atmospheric Sounding Interferometer New Generation (IASI-NG) is a key payload element of the second generation of European meteorological polar-orbit satellites (METOP-SG).

It will continue and improve the IASI mission in the next decades (2020-2040) in the field of operational meteorology, climate monitoring, and characterization of atmospheric composition related to climate, atmospheric chemistry and environment. The main challenge is the performance improvement by a factor two compared to IASI with regard to spectral resolution and radiometric error.

The measurement technique is based on wide field Fourier Transform Spectrometer (operating in the 3.5 - $15.5 \mu \mathrm{m}$ spectral range) with an innovative Mertz compensated interferometer to manage the so-called self-apodisation effect and the associated spectral resolution degradation.

End 2019, IASI-NG instrument Critical Design Review has been successful and since nearly all the sub-units of the PFM instrument have been fully characterized and delivered to ADS. Interferometer principle has been partially validated on a Bread Board Model and Interferometer EQM and PFM have completed qualification and performance test.

The aim of this paper is first to present the performance budget of the instrument after CDR, based on sub-units consolidation (sub-unit CDR) or measurement (sub-assemblies PFM measurement). We will then focus on the overall instrument development status and the different upcoming milestones until the delivery.
\end{abstract}

Keywords: infrared, Fourier Transform Interferometer, Mertz, KBr, METOP-SG, NedT, ISRF

\section{INTRODUCTION}

IASI NG (Infrared Atmospheric Sounding Interferometer New Generation) is on-board of Metop-SG Satellites developed by ESA and part of EPS-SG, developed by Eumetsat in order to extend the mission EPS for the next decades (2020-2040). CNES is in charge of the overall IASI-NG system, namely Instruments, Ground Processing Software, Technical Expertise Center. The 3 Flight Models of the instrument are manufactured by Airbus Defence \& Space. IASI-NG shall provide operational meteorology data such as atmospheric temperature and $\mathrm{H} 2 \mathrm{O}$ vapor profiles as well as other gases monitoring like ozone, methane, carbon monoxide [3]

IASI-NG Level1-product delivered by the IASI-NG system are geo-localized, spectrally and radiometrically calibrated spectra. IASI-NG instrument is composed by an IR-imager and an IR-sounder, and the performances of the sounder only are explained in this paper. Main specifications are recall in Table1. Regarding geometry and spectral coverage, the specifications are identical to the IASI ones in order to ensure mission continuity. For radiometric noise and calibration as well as spectral sampling, resolution and calibration, the performances goals are two times better than IASI, leading to an instrumental concept change [1] in, order to improve the instantaneous field of regard (FOR) of the sounder. So the FOR of IASI-NG is composed by a $4 \times 4$ sounder pixels pattern in comparison of a $2 \times 2$ sounder pixels for IASI (Fig1); and the interferogram acquisition time is about $0.74 \mathrm{~ms}$ for IASI-NG. 
Table 1. IASI-NG main requirements

\begin{tabular}{|l|l|l|}
\hline Parameter & Requirement & Value \\
\hline Geometry & sounding point size & $\approx 12 \mathrm{~km}$ \\
& spatial sampling & $\approx 25 \mathrm{~km}$ \\
& geolocation error & $0.5 \mathrm{~km}$ \\
\hline Radiometry & radiometric calibration error & $0.25 \mathrm{~K} @ 280 \mathrm{~K}$ \\
& NedT@280K & 0.1 to $0.4 \mathrm{~K}$ within the spectrum \\
\hline Spectral & continuous spectral covering & From $3.6 \mu \mathrm{m}$ to $15.5 \mu \mathrm{m}$ \\
& spectral resolution & $0.25 \mathrm{~cm}^{-1}$ \\
& spectral sampling & $0.12 \mathrm{~cm}^{-1}$ \\
& spectral calibration error & $510^{-7}$ \\
\hline
\end{tabular}

\section{DESIGN AND PERFORMANCES DRIVERS}
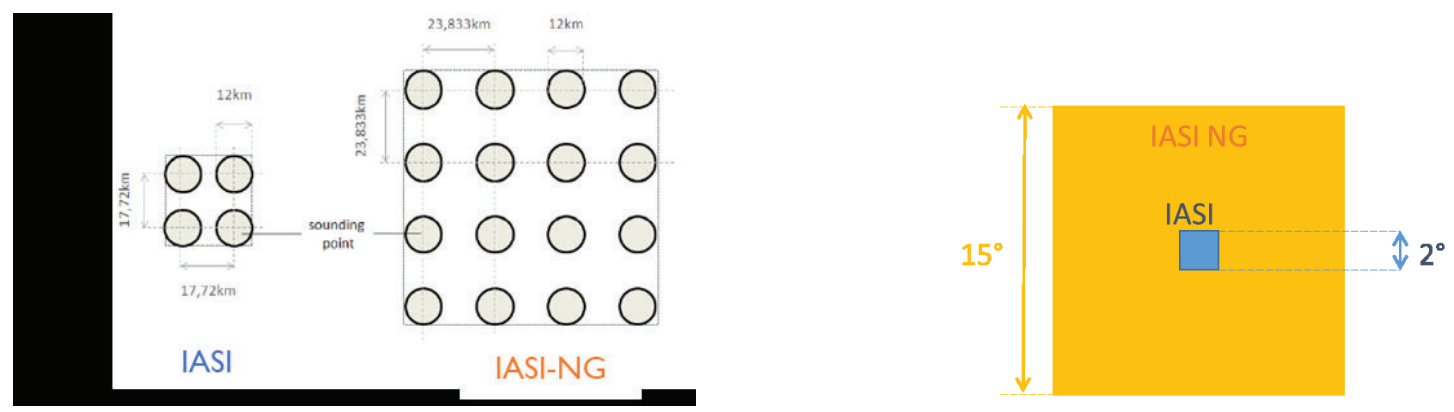

Figure 1. Scheme of the Field of regard on Earth (left) and inside the interferometer (right): IASI/ IASI-NG comparison.

\subsection{Mertz Interferometer}

The detailed design of Mertz interferometer has been exposed in [4] and is sketched in Fig 2.

The Mertz Interferometer is composed by two pairs of prisms: the internals and the externals prims that have a reflecting face. As in a Michelson interferometer, the incoming optical flux is separated in amplitude by the separating plate, associated with a compensating plate for symmetry. Moving the two pairs of prism with the translations indicated by the arrows, allows to tune in the same time the difference of the mirrors positions and the difference of the blade (formed by the Internal-external prisms) thickness between the two arms.

The optical path difference OPD can be written as

$$
O P D=2\left[\Delta e_{\text {glass }} \sqrt{n^{2}-\sin ^{2} \theta}-\Delta e_{\text {air }} \cos \theta\right]
$$

where $\Delta e_{\text {glass }}$ stands for the difference of glass thickness between the two arms of the interferometer, $\Delta e_{\text {air }}$ the difference of the air thickness, $\mathrm{n}$ the refractive index of the prism material : $\mathrm{KBr}$ for IASI-NG design, and $\theta$ the field angle inside the interferometer.

This equation becomes quite independent of the field angle $\theta$ when the Mertz compensation condition is fulfilled : $\Delta e_{\text {air }}=$ $-\frac{\Delta e_{\text {glass }}}{n}$. This condition is a proportionality between differential air thickness and differential glass thickness, including the glass refraction index. It will be exactly fulfilled only for a specific wavelength, but the residues over the wide IASING spectral band are negligible. This compensation allows to widen the field and reduce the pupil diameter inside the interferometer as explained in Fig 1 (right).

In practice the prisms are moved by a single mechanism called the dual swing mechanism, patented by ADS. This mechanism introduces two rotating translations with a fixed proportionality in order respect the compensation condition. It is represented on Fig 2 (right). 

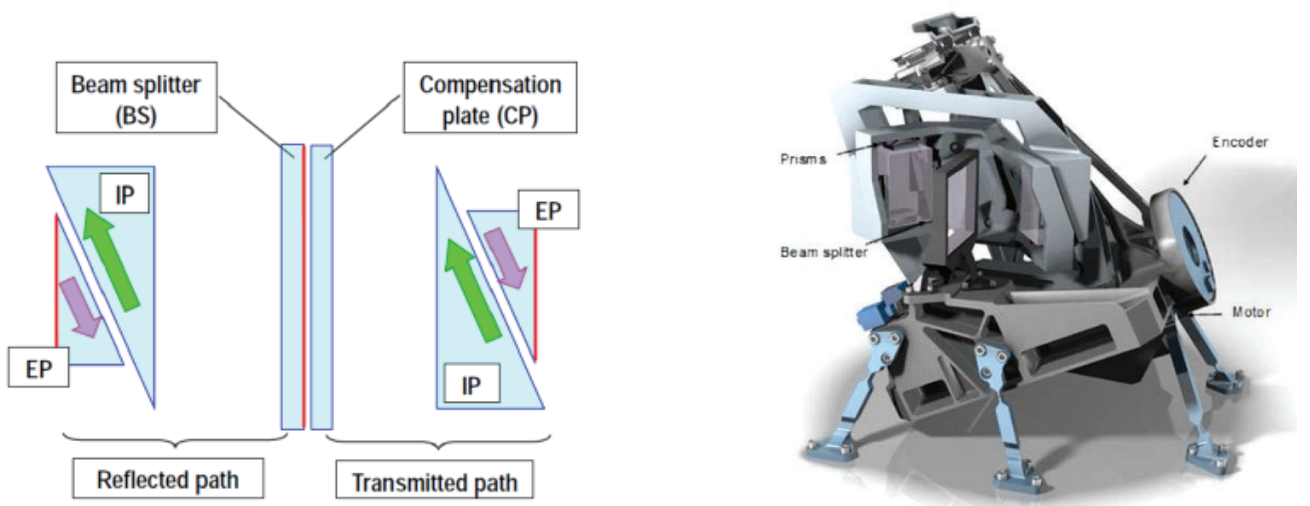

Figure 2. (left) sketch of the Mertz Interferometer (right) Mertz Interferometer design with Dual Swing Mechanism for IASI NG instrument

The benefits of this design is that the interferogram envelop (called Modulation Efficiency or ME in the paper) and phase (and so the effective Optical Path Difference OPD) are quite independent of the field, reducing drastically the autoapodisation effect that limits the Michelson interferometer in similar conditions of field and arm length. Field effects being negligible, the pupil's effects are predominant on the shape of the ME and OPD and become a performance driver. The differential wave front errors in the pupil plane is usually decomposed between tilt (first order Zernike polynomial decomposition) and higher orders named WFE in this paper. Let us detail the consequences of both effects.

A tilt leads at first order to a loss of contrast driven by the well-known formula

$$
\operatorname{ME}(\alpha(t))=\frac{J_{1}\left(\pi \sigma \alpha(t) D_{\text {pup }}\right)}{\pi \sigma \alpha(t) D_{\text {pup }}}
$$

with $\sigma$ the wavenumber, $\alpha$ the differential tilt, $D_{\text {pup }}$ the pupil diameter inside the Interferometer. In order to optimize the contrast, the beam splitter assembly can be realigned on ground and in flight over $500 \mu \mathrm{rad}$. This realignment ensures a very good spectral performance, but concern only long terms drifts. Short term variation, especially microvibrations inside the interferometer are critical.

The WFE sensitivity is approximated by the formula

$$
M E(t) \sim 1-2\left(\pi \sigma \delta W F E_{r m s}(t)\right)^{2}
$$

with $\delta W F E_{r m s}$ the differential surface error cumulated by the science beam crossing the blades and the prisms surfaces. As the prisms are moving, this quantity is time-dependent and cannot be ignored in the instrument modelization.

As can be noticed in the OPD simplified formula (1), the OPD is deeply linked to the refractive index of the prisms and so to the wavenumber. As the consequence the spectral calibration is wavenumber dependent with a variation linked to the refractive index difference between metrology wavenumber and science wavenumber (Fig 6). This instrument requires a specific ground and flight calibration strategy in order to comply with the mission requirements.

\subsection{Instrumental design}

The instrumental design is detailed in Fig 3 and reference [2]. The main performances drivers are recalled hereafter.

We just have seen that the Mertz interferometer allows to acquire $4 \times 4$ sounder Pixel at the same time, improving acquisition duration up to $0.74 \mathrm{~s}$ that benefits to the SNR as well as the spectral performances.

Inside the interferometer, a 5-beams metrology at $1.5 \mu \mathrm{m}$ allows to monitor precisely and in real time the OPD and the prisms differential rigid body behavior, with a decomposition on the 4 first Zernike polynomials: tilt_u, tilt_v, focus and astigmatism. 
Around the interferometer, the optical flux from $3.6 \mu \mathrm{m}$ to $15.5 \mu \mathrm{m}$ is split in 4 bands in the focal plane assembly and is detected with high performance cooled IR detectors (PC for B1, PV, for B2 B3 B4). The B1-detector temperature of $75 \mathrm{~K}$ is ensured with active cryo-cooling.

The geometric performances are optimized at both side of the optical chain: on one side the scan mirror and its mechanism pointing performances, on the other side, the optical design of the Cold Optics optimized for IPSF geometric performances.

Regarding radiometric calibration as for IASI a high performance warm blackbody is measured at each end of swath as well as a cold space view.

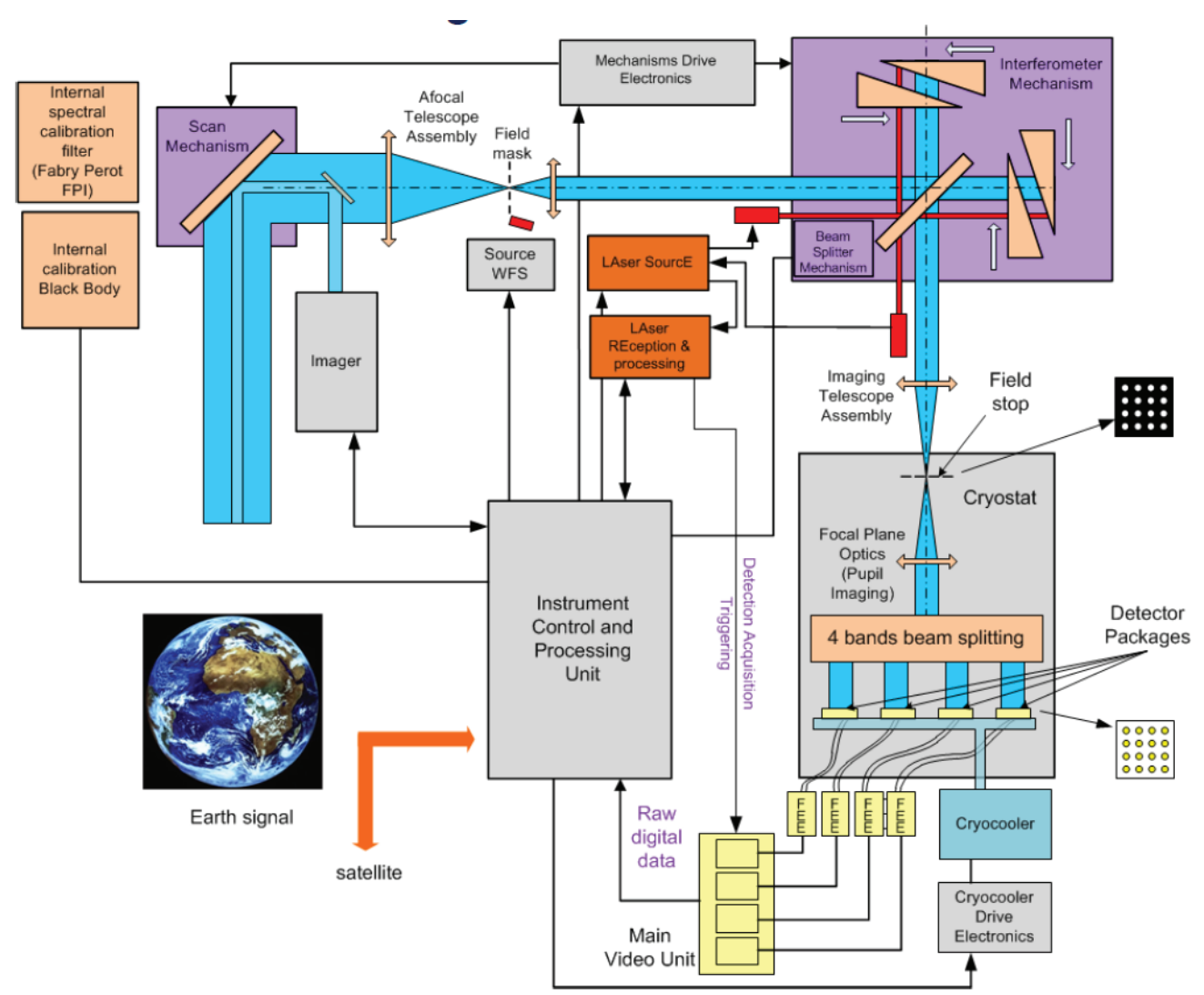

Figure 3. (left) sketch of the Mertz Interferometer (right) Mertz Interferometer design with Dual Swing Mechanism for IASI NG instrument

The new instrumental concept includes two calibration sources that where not present for IASI: a Fabry-Perot view for spectral calibration monitoring and a WFS source at $3.6 \mu \mathrm{m}$ for spectral performances and model long term monitoring.

\section{GEOMETRY}

The geometric requirements concern first the ground positioning of the sounder pixel and second the relative radiometric transmission inside the sounder pixel.

Concerning the ground positioning of the sounder pixel: the sampling grid represented in fig 4 is slightly irregular with a maximal error of $3.2 \mathrm{mrad}$ for a specification of $3 \mathrm{mrad}$. This is mainly due to the optical distortions in the TMA telescopes. With a knowledge of $0.2 \mathrm{mrad}$ (spec $0.2 \mathrm{mrad}$ ) and a repeatability of $0.5 \mathrm{mrad}$ ( $\mathrm{spec} 1 \mathrm{mrad}$ ) the performance is considered achieved. The pointing stability of $62 \mu \mathrm{rad}(\mathrm{spec} 100 \mu \mathrm{rad})$ and its accuracy of $0.64 \mathrm{mrad}$ ( $\mathrm{spec} 3 \mathrm{mrad}$ ) are well within 
specification. The imager sounder co-registration is out of spec mainly due to the ground to flight setting and becomes compliant considering a flight calibration during calval phase.

The IPSF specifications evaluations are gathered in Tables 2, 3 and 4. Table 2: the IPSF uniformity is well within the $20 \%$ specification for all sounder pixels and the four spectral bands. Table 3 refers to the crosstalk performances measuring the flux coming from other pixels considered as a pollution. The expected performance is within the specification for all bands but for B1-band where ghost contributions is the main contributor. In table 4 the expected result of the far field specification is presented. With a budget compliance depending on the wavelength as this performance is mainly linked to scattering effects due to contamination in the analysis. At system level all this partial non-conformity is taken into account to get the system budget, which is discussed with IASI-NG users.

Table 2. IASI-NG IPSF uniformity budget at CDR

\begin{tabular}{|c|c|c|c|c|}
\hline Sounder Pixel & B1 & B2 & B3 & B4 \\
\hline \#1 (corner) & $6.0 \%$ & $8.8 \%$ & $7.2 \%$ & $3.5 \%$ \\
\hline$\# 6$ (center) & $5.7 \%$ & $6.7 \%$ & $11.6 \%$ & $6.5 \%$ \\
\hline$\# 8$ (side) & $5.9 \%$ & $4.3 \%$ & $14.7 \%$ & $10.7 \%$ \\
\hline$\# 16$ (comer) & $5.6 \%$ & $5.3 \%$ & $14.2 \%$ & $10.8 \%$ \\
\hline Specification & \multicolumn{3}{|c|}{$\mathbf{2 0} \%$} \\
\hline
\end{tabular}

Table 3 IASI-NG Cross Talk budget at CDR

\begin{tabular}{|l|c|c|c|c|}
\multicolumn{1}{|c|}{ Crosstalkc } & B & B2 & B3 & B4 \\
\hline TOTAL :Average pixel & $0.39 \%$ & $0.21 \%$ & $0.22 \%$ & $0.22 \%$ \\
\hline TOTAL :Worst case pixel & $0.45 \%$ & $0.25 \%$ & $0.28 \%$ & $0.28 \%$ \\
\hline Specification & \multicolumn{4}{|c|}{$0.3 \%$} \\
\hline
\end{tabular}

Table 4 IASI-NG Far Field budget at CDR

\begin{tabular}{|l|c|c|}
\multicolumn{1}{|c|}{ Far Field } & BI & B4 \\
\hline Far field illumination - scan @ I600 ppm & $0.21 \%$ & $0.42 \%$ \\
\hline Far field illumination - scan @ 2040 ppm & $0.26 \%$ & $0.53 \%$ \\
\hline Specification & \multicolumn{2}{|c|}{$0.3 \%$} \\
\hline
\end{tabular}

\section{SPECTRAL}

\subsection{Ground processing correction}

Spectral performances aim at quantifying the difference between an ideal ISRF (Gaussian) and the measured ISRF: these differences are expressed in terms of centroid shift, shape variations and parasites for microvibration. As the expected performances are really stringent, a ground processing correction can be taken into account, and the performance is estimated after correction.

The ground processing goal is to calculate in real time with 5 beams metrology OPD telemetry the science error OPD, $\widehat{\delta O P D}(\mathbf{m e t}+\mathbf{c h a r a c})$, and the science modulation efficiency, $\widehat{\mathrm{ME}}(\boldsymbol{m e t}+\boldsymbol{c h a r a c})$. This calculation is not simplified as equations (2) and (3) approximations for the Modulation Efficiency, but take into account the defects presented in paragraph 2: tilts due to mechanisms defects or vibrations, WFE due to prisms surface errors, KBr refraction index as well as science and metrology geometry inside the interferometer. So the model is wavenumber and pixel dependent and leans on precise ground characterizations. 
Thanks to this ground model, sort of a deconvolution is made and the final response is very similar to the expected Gaussian response with regular OPD. The spectral performances are estimated on the FFT of the corrected interferogram $I F G^{0 r}$ defined by :

$$
I F G^{0 r}=\mathrm{ME} \cdot e^{2 i \pi \sigma \cdot(\mathrm{OPD}+\delta \mathrm{OPD})} \times \frac{1}{\widehat{\mathrm{ME}(\text { met }+ \text { charac })}} \cdot e^{-2 i \pi \sigma \cdot \delta \widehat{\mathrm{OPD}}(\text { met }+ \text { charac })} \times G^{T F r e f} \sim G^{T F r e f} e^{2 i \pi \sigma O P D}
$$

- The first term of the product represents the monochromatic measure (sine complex function) of the real instrument.

- The second term represents the inverse of the model estimation (sine complex function too).

- The third term is the Gaussian apodisation.

In order to estimate the spectral performances, a set of instrument defects and mis-knowledges has been randomly chosen and interferogram simulations including correction process has been performed by ADS.

\subsection{Shape and centroid stability performances}

The results of the set of instruments trials are given in Fig4 for the centroid stability and shape error index.
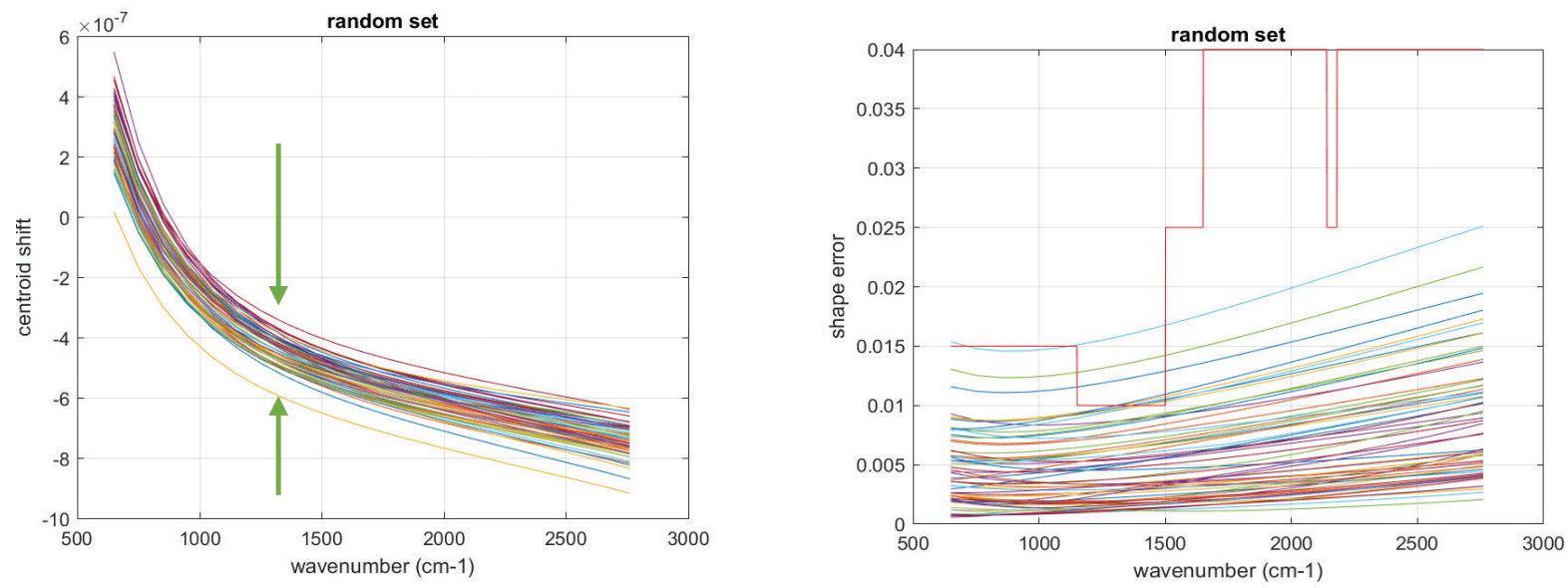

Figure 4. (left) Centroid stability over orbit simulation. (right) Shape error index simulations (red plot = specification) for IASI NG instrument at CDR

The centroid shift stability is within the specification of $5.10^{-7}$. The performance is met for the shape error index, in the great majority of the simulated cases.

\subsection{Microvibration performances}

Considering now the spectral specification linked to the microvibrations inside the interferometer

Microvibrations $(20 \mathrm{~Hz}$ up to $200 \mathrm{~Hz})$ inside the interferometer creates ME modulation as well as OPD modulation. For each vibration frequency $f$, a pair of parasites appears around the main peak of the ISRF at $+/-f$ /Vopd wavenumber. These parasites are specified in relative amplitude to the main peak in the instrument specification.

Estimating the amplitude of these peaks, requires to first evaluate the vibrations frequencies and amplitudes inside the interferometer and then simulate science and metrology ME and OPD inside the interferometer and take into account ground processing. The mechanical analysis has been carried with up-to-date microvibration sources hypothesis for IASING instrument as well as the other satellites contributors (METIMAGE, MWS, 3MI, the wheels). And a mechanical coupled model of the platform and the instruments is used to get realistic mechanical transfer-functions between the sources and the interferometer components. After this mechanical analysis the displacement magnitude is deduced for the prisms 
and blades inside the interferometer: the vibrations are about $5 \mu \mathrm{rad}$ in rotation and $100 \mathrm{~nm}$ in translation. The most perturbed element is the beam-splitter and the main perturbator is IASI-NG cryo-cooler.

With the mechanical displacements an optical simulation allows to simulate the science acquisition and the metrology ones, and to mimic the correction process.

The results are presented in the table Fig 5 for the OPD vibration and the ME Vibration. The single harmonic budget is compliant over a large spectral domain and becomes non-compliant at short wavenumber. The budget made with an absolute sum on contributors and frequencies is out of specification. The system budget has to take into account each frequency, amplitude couple to assess the impact on the mission performances.

As the whole process of evaluation includes several margin and hypothesis to be consolidated, this NC leads to specifics verification activities in the phase $\mathrm{D}$ in order to reduce the margin errors and confirm the real performances. Moreover, activities on the ground model have to be done in order to optimize the microvibration correction process.

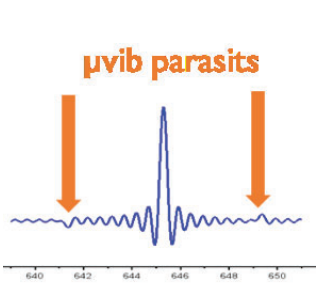

\begin{tabular}{|c|c|c|c|c|}
\hline \multirow{2}{*}{$\begin{array}{c}\text { Microvibration } \\
\text { Performances }\end{array}$} & \multicolumn{2}{|c|}{ OPD vibration } & \multicolumn{2}{|c|}{ ME vibration } \\
\hline Spec & $\begin{array}{r}650 \mathrm{~cm}^{-1} \\
15,5 \mu \mathrm{m}\end{array}$ & $\begin{array}{r}2760 \mathrm{~cm}^{-1} \\
3,62 \mu \mathrm{m}\end{array}$ & $\begin{array}{r}650 \mathrm{~cm}^{-1} \\
15,5 \mu \mathrm{m}\end{array}$ & $2760 \mathrm{~cm}^{-1}$ \\
\hline Single harmonic budget & $0,25 \%$ & $0,25 \%$ & $0,025 \%$ & $0,025 \%$ \\
\hline $\begin{array}{c}\text { Total budget (sum on } \\
\text { harmonics and contributors) }\end{array}$ & $0,07 \%$ & $0,30 \%$ & $0,002 \%$ & $0,040 \%$ \\
\hline
\end{tabular}

Figure 5. (left) microvibration impact on ISRF. (right) Microvibration performances estimated at CDR, the impact of the ground processing being partially taken into account.

\subsection{Spectral calibration performances}

As explained in paragraph 2.1., IASI-NG centroid depends strongly of the wavenumber. The spectral "de-calibration" after FFT before any correction process is between $21 \mathrm{~cm}^{-1}$ at $645 \mathrm{~cm}^{-1}(15.5 \mu \mathrm{m})$ and $11 \mathrm{~cm}^{-1}$ at $2760 \mathrm{~cm}^{-1}(3.6 \mu \mathrm{m}) \mathrm{see} \mathrm{Fig} 6$. The a-priori knowledge of the spectral law at $3.410^{-5}$ is achieved thanks to the refractive index measurements and science and metrology beams geometry inside the interferometer characterization.

The final spectral calibration at $10^{-6}$ at system level is achieved in flight thanks to a complex calibration process on atmospheric spectra measured by the instrument (ref [3], [5]). A verification of the smooth behavior of the spectral law can be done with Fabry-Perot acquisitions, that presents regular oscillations over the whole IASI-NG (Fig 7. right). This process lean on the good centroid stability of the instrument as expected in paragraph 4.2.

A tight spectral characterization and verification is foreseen at instrument thermal vacuum with a gas cell as well as internal Fabry Perot (FPI) acquisitions. The whole process will be done and its performances checked.
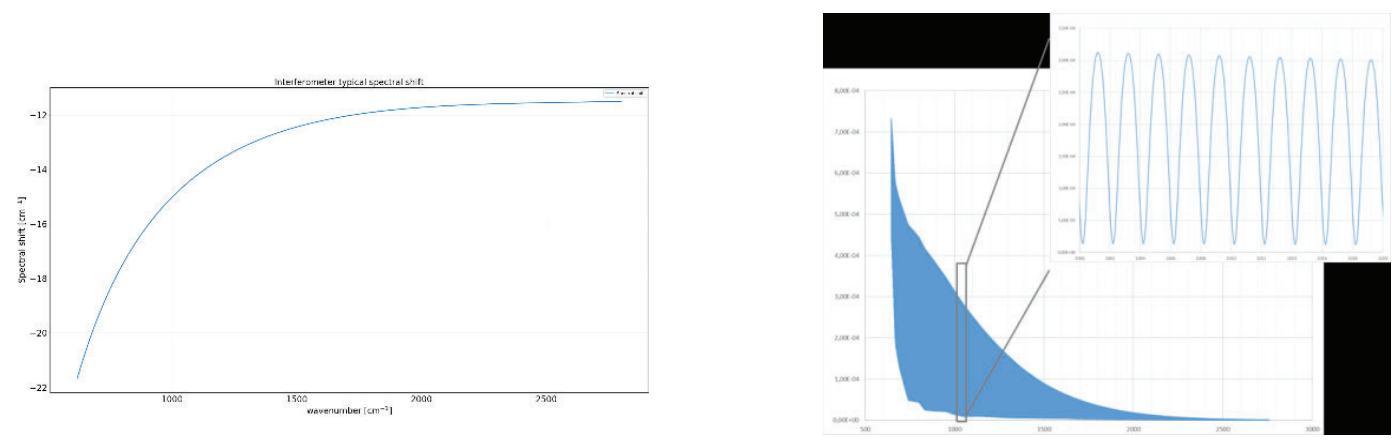

Figure 6. (left) spectral native de-calibration of IASI-NG. (right) Fabry Perot simulated spectra 


\section{RADIOMETRY}

\subsection{Absolute calibration}

The Fig 7 represents the absolute calibration error budget over the whole lifetime as a function of the wavenumber. The several colors correspond to the equivalent blackbody scene at several temperatures: from one hundred eighty Kelvin to three hundred and ten Kelvin.

In this budget two kinds of contributors are considered:

- The first ones are linked to the on board Blackbody and the errors do to the knowledge on its emissivity or exact temperature.

- The second ones aim to quantify the differences between the earth views and the calibrations views like the background instabilities, the gains instabilities, non-linearity residuals, differential straylight.

This overall budget takes into account different timescale contributors short and long terms. The estimated budget at CDR is compliant with margins.

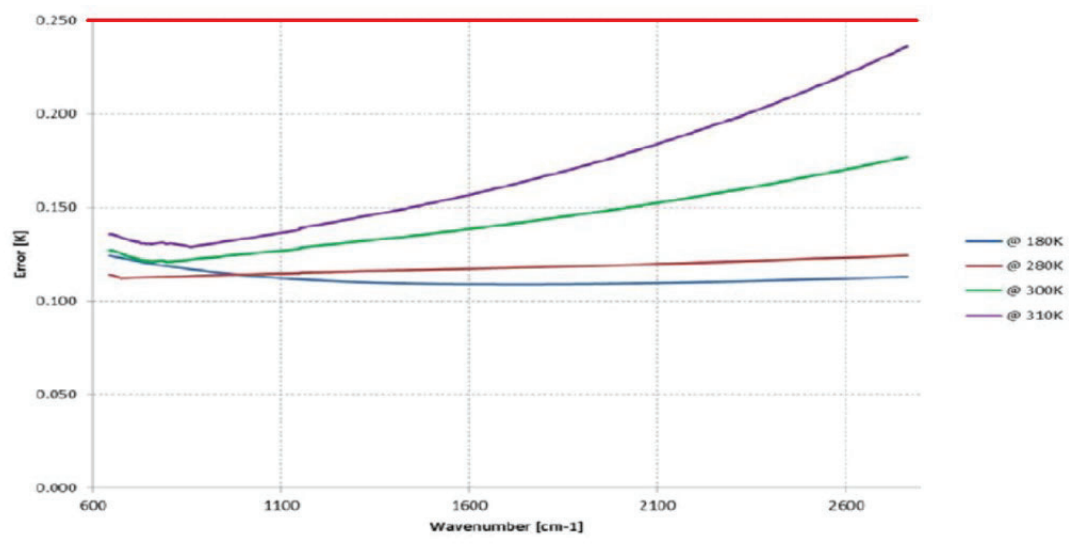

Figure 7. absolute radiometric calibration performance budget

\subsection{NedT performance}

The plot here represents the NedT budget for a blackbody scene at $280 \mathrm{~K}$. The budget includes some ground processing contributors linked to the spectral correction process explained in paragraph 4.1. The sixteen colored plot correspond to the sixteen sounder pixel. The NedT performance is expected be very good in general for all spectral bands, especially the $\mathrm{B} 1$ thanks to the good $\mathrm{KBr}$ transmission and optimized broad band optical coatings.

The slight non conformances are in line for system budgets. 


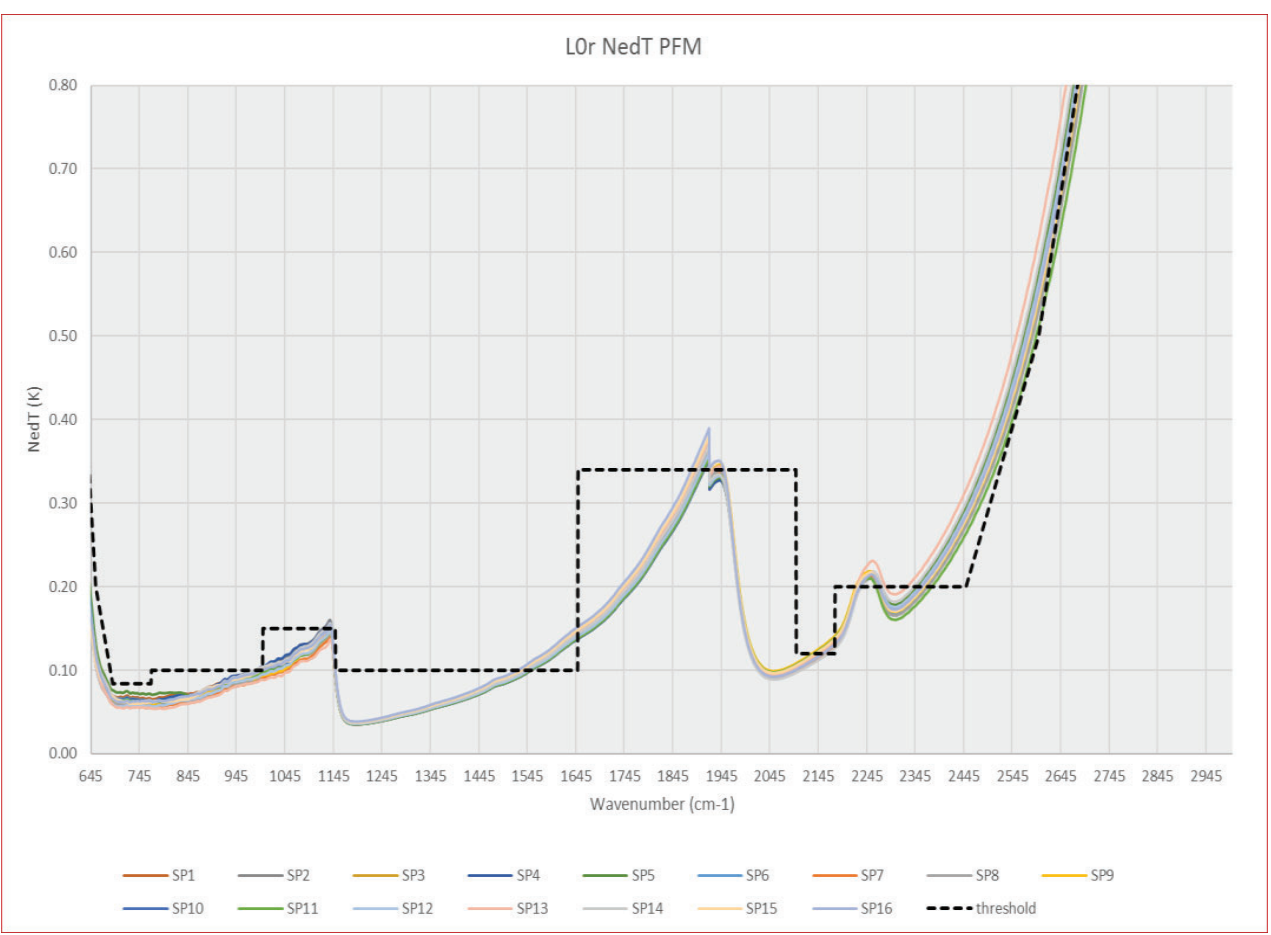

Figure 8. radiometric noise performance budget for à Black Body at $280 \mathrm{~K}$, including some ground processing contributors and filtering

\section{CONCLUSIONS, TOWARD PERFORMANCES VERIFICATION}

Regarding performances estimation, the CDR status is in line with the specification. Microvibrations performances is a concern and a consolidation of the CDR evaluation is in progress thanks to realistic measurements in EQM interferometer model and EM instrument in order to reduce the uncertainties on the mechanical sources and transfer functions. The optimization of the correction process is in progress, and rely on real measurement of PFMinterferometer.

Performance verification is currently on-going at ADS at subsystem level [1]. The first spectral measurements of the Mertz interferometer with PFM model has been done for one sounder pixel in order to verify the centroid stability and its thermal sensitivity, the shape error index and microvibration sensitivity with respect to the modelization. At focal place assembly, the main contributors of the geometric transmission inside the sixteen sounders pixels, and a measure of the crosstalk has been performed as well as a detectors noise and responsivity checks in the 4 bands.

The main test to come is the instrument thermal vacuum for radiometric and spectral performances assessment. For the radiometric measurements, two high performances blackbodies with temperature varying from $200 \mathrm{~K}$ to $310 \mathrm{~K}$ associated with a cold space view at $80 \mathrm{~K}$ are foreseen. On the spectral performances side, the shape and centroid stability will be assessed with 4 IR-lasers (one per band). As explained in paragraph 4.4 the spectral calibration measurement will be the internal Fabry-Perot and gas cell. All the performances will be measured within the year for PFM model.

\section{REFERENCES}

[1] Calvel, B., Bernard, F., "IASI-NG development status”, Proc. ICSO Conference, Oct. 2018. 
[2] Calvel B., Penquer A. "IASI-NG development status", Proc. ICSO Conference, Mars. 2021.

[3] A. Deschamp "IASI-NG development status", Proc. ICSO Conference, Mars. 2021.

[4] C. Luitot, J. Boyadjian, C. Buil, F. Pasternak "Optical architecture of the new generation infrared atmospheric sounder interferometer (IASI-NG)" 25 September 2013 Proc. SPIE Optical Engineering, Volume 8841, Current Developments in Lens Design and Optical Engineering XIV; 88410M (2013) https://doi.org/10.1117/12.2025236

[5] A. Deschamps, C. Luitot, E. Baldit, A. Penquer, L. Watson, N. Coutand, "IASI-NG Level1 processing : how to estimate the instrument spectral response in real-time", $22^{\text {nd }}$ International TOVS Study Conference, 2019, http://cimss.ssec.wisc.edu/itwg/itsc/ 